\title{
Influence of FTO variants on obesity, inflammation and cardiovascular disease risk biomarkers in Spanish children: a case-control multicentre study
}

Josune Olza ${ }^{1}$, Azahara I Ruperez ${ }^{1}$, Mercedes Gil-Campos², Rosaura Leis ${ }^{3}$, Dietmar Fernandez-Orth ${ }^{4}$, Rafael Tojo ${ }^{3}$, Ramon Cañete ${ }^{2}$, Angel Gil ${ }^{1}$ and Concepcion M Aguilera ${ }^{1 *}$

\begin{abstract}
Background: Variants in the FTO gene have been associated with obesity in children, but this association has not been shown with other biomarkers. We assessed the association of 52 FTO polymorphisms, spanning the whole gene, with obesity and estimated the influence of these polymorphisms on anthropometric, clinical and metabolic parameters as well as inflammation and cardiovascular disease (CVD) risk biomarkers among Spanish children.

Methods: A multicentre case-control study was conducted in 534 children (292 obese and 242 with normal-BMI). Anthropometric, clinical, metabolic, inflammation and CVD risk markers were compared using the Student's t-test for unpaired samples. The genotype relative risk was assessed by comparing the obese and normal-BMl group, calculating the odds ratio. The association of each SNP with phenotypic parameters was analysed using either logistic or linear regression analysis.

Results: All anthropometric, clinical and metabolic factors as well as inflammatory and CVD risk biomarkers were higher in the obese than in the normal-BMl group, except adiponectin and HDL-c that were lower, and glucose, LDL-C, and metalloproteinase-9 that did not show difference. Four polymorphisms (rs9935401, rs9939609, rs9928094 and rs9930333) were positively associated with obesity and in linkage disequilibrium between each other; the haplotype including the risk alleles of these polymorphisms showed a high risk for obesity. The rs8061518 was negatively associated with obesity and the haplotype including this SNP and rs3826169, rs17818902 and rs7190053 showed a decreased risk for obesity. Additionally, the rs8061518 was associated with weight, diastolic blood pressure, insulin, homeostatic model assessment of insulin resistance, leptin, and active plasminogen inhibitor activator-1 after sex and age adjustment; however, after an additional BMI adjustment, this polymorphism remained associated only with leptin.

Conclusions: We validated the previous reported association of genetic variability in intron 1 of the FTO gene with the risk of obesity and found no association with other related traits in this region of the gene. We have observed strong statistical evidence for an association of rs8061518 in intron 3 of the gene with decreased risk of obesity and low concentration of leptin.
\end{abstract}

Keywords: FTO, Genetic polymorphism, Obesity, Child, Inflammation, CVD

\footnotetext{
* Correspondence: caguiler@ugr.es

'Department of Biochemistry and Molecular Biology II, Faculty of Pharmacy Institute of Nutrition and Food Technology, University of Granada, Granada, Spain

Full list of author information is available at the end of the article
} 


\section{Background}

Obesity is described as a disease with genetic and environmental influence that may lead to several comorbidities such as type 2 diabetes, cardiovascular diseases (CVD), metabolic syndrome (MS) and respiratory abnormalities among others $[1,2]$. Since the discovery of its association with variants in intron 1 of the fat mass and obesity associated (FTO) gene [3-5], many studies have been conducted to replicate this finding, to identify new variants, to clarify the biological function of the protein encoded and to explain its role in the origin and/or development of obesity [6]. FTO was initially associated with type 2 diabetes mellitus in European descent population [3]; however, simultaneous studies showed that the association was mediated by its effect on obesity $[4,5]$. This association with obesity also has been observed in different populations as Europeans [4,5,7-9], Asians [10,11], African Americans [12,13] and Hispanic Americans [13]. Among the identified polymorphisms, the rs9939609 is one of the most strongly associated with common obesity $[10,14,15]$; however, several other single nucleotide polymorphism (SNP)s in the first intron of the FTO gene that are in linkage disequilibrium (LD) with rs9939609 showed similar effects on both childhood $[4,7,16]$ and adult obesity $[3,4]$.

It is common to observe clinical, metabolic and CVD risk factors in many, but not all obese children. However, few studies include a large number of biomarkers of inflammation and CVD risk in the paediatric population and compare the levels of these markers with the presence or absence of genetic variants. For this reason, we assessed the association of 52 SNPs spanning the FTO gene, with obesity in Spanish children; we also estimated the influence of these SNPs in anthropometric, clinical and metabolic parameters as well as inflammatory and CVD risk markers.

\section{Methods \\ Study design}

The present study was designed as a case-control multicentre study in children. We recruited 534 children, 292 classified as obese according to body mass index (BMI), using the age- and sex-specific cut-off points of Cole et al. [17] (149 boys and 143 girls), and 242 as normal-BMI (135 boys and 107 girls). The children were prepubertal and pubertal aged 6-15 years and were recruited in two cities of Spain (Cordoba and Santiago de Compostela) from primary care centres and schools. Inclusion criterions were European descent and the absence of congenital metabolic diseases. Exclusion criterions were non-European descent, the presence of congenital metabolic diseases, for example, diabetes or hyperlipidaemia, or undernutrition and the use of medication that alters blood pressure (BP) and glucose or lipid metabolism. After assessments made on a first visit to the school or primary care centre, the parents of children fulfilling the inclusion criteria were invited to take the children to the paediatrics unit of the participating hospitals for a clinical examination.

\section{Ethics statements}

The protocol was performed in accordance with the Declaration of Helsinki (Edinburgh 2000 revised) and following the recommendations of the Good Clinical Practice of the CEE (Document 111/3976/88 July 1990) and the in force law of the Spanish regulation, that regulates Clinical Trials in human beings (RD 223/04 about Clinical Trials). The Ethics Committee on Human Research of the University of Granada, the Ethics Committee of the Reina Sofía University Hospital of Cordoba and the Bioethics Committee of the University of Santiago de Compostela approved it. Written informed consent was obtained from parents of children, and all children gave their assent.

\section{Anthropometric and biochemical measurements}

Anthropometric measurements were taken by a single examiner with the children barefoot and in their underwear. Body weight $(\mathrm{kg})$ and height $(\mathrm{cm})$ were measured using standardized procedures, and waist circumference (WC) was measured midway between the lowest rib and the superior border of the iliac crest on standing subjects, using an inelastic measuring tape at the end of normal expiration. BMI was calculated as weight $(\mathrm{kg})$ divided by the square of the height $\left(\mathrm{m}^{2}\right)$. BP was measured three times by the same examiner, using a mercury sphygmomanometer and following international recommendations. Blood samples were drawn via the antecubital vein after the patient had fasted overnight. Insulin resistance was calculated by means of the homeostatic model assessment of insulin resistance (HOMA-IR). General biochemical analyses were performed at the participating University Hospital Laboratories following internationally accepted quality control protocols.

\section{Cardiovascular and inflammatory biomarkers}

Three different LINCOplex ${ }^{\mathrm{TM}}$ kits of human monoclonal antibodies (Linco Research, St. Charles, MO) were used on a Luminex $200^{\mathrm{TM}}$ System (Luminex Corporation, Austin, TX) to determine: 1) adiponectin (coefficient of variation $[\mathrm{CV}]: 7.9 \%)$, resistin $(\mathrm{CV}: 6.0 \%)$ and active plasminogen activator inhibitor-1 (PAI-1) (CV: 6.6\%) (Cat. HADK1-61 K-A); 2) interleukin (IL)-6 (CV: 7.8\%), IL-8 (CV: 7.9\%), leptin (CV: 7.9\%) and tumour necrosis factor alpha (TNF- $\alpha$ ) (CV: 7.8\%) (Cat. HADK2-61 K-B); and 3) soluble intercellular adhesion molecule 1 (sICAM-1) (CV: 7.9\%), soluble endothelial selectin (sE-selectin) (CV: $11.2 \%)$, myeloperoxidase (MPO) (CV: 12.3\%), matrix metalloproteinase 9 (MMP-9) (CV: 6.8\%) and total PAI-1 (CV: 11.8\%) (Cat. HCVD1-67 AK). C reactive protein 
(CRP) (CV: 4\%) was determined with a particle-enhanced turbidimetric immunoassay (PETIA) (Dade Behring Inc., Deerfield, IL).

\section{DNA isolation and genotyping}

Genomic DNA was extracted from peripheral white blood cells (buffy coat) using the QIAamp Blood kit (Qiagen, Valencia, CA) according to the manufacturer instructions. 52 SNPs in the FTO gene were selected. SNPs eligibility was based on their location, first selecting every missense variation and then others located in the promoter, 3'UTR and $5^{\prime}$ UTR regions with a minor allele frequency (MAF) higher than 0.05 in the European descent population from the NCBI database and a minimum pair wise LD of $r^{2}=$ 0.8 for the selection of TagSNPs from the HapMap. In addition, SNPs described to be positively associated with obesity in relevant publications were also included. Genotyping was performed by the Illumina GoldenGate Assay (Illumina Inc., San Diego, CA) on 96-well format Sentrix ${ }^{\circ}$ arrays. Two hundred fifty nanograms of DNA samples were used per assay. Genotyping of the 52 SNPs resulted in a success rate $>97 \%$. Five SNPs (rs1125337, rs9929152, rs16952649, rs8053966 and rs8054364), which deviated from Hardy-Weinberg equilibrium in the normalBMI group $(\mathrm{p}<0.05)$ were excluded from the analysis (Additional file 1: Table S1). Because our population proceeds from two different cities of Spain, we performed a meta-analysis with the two populations as independent groups to avoid problems like population stratification or genotyping batch effect. The result of this analysis ( $p$ values of Q Cochrane: rs9939609 Q $=0.967$, rs9935401 $\mathrm{Q}=0.888$, rs9930333 $\mathrm{Q}=0.795$, rs9928094 $\mathrm{Q}=0.867$ and rs8061518 $\mathrm{Q}=0.926$ ) indicate little detectable heterogeneity for the two considered populations of the study.

\section{Statistical analysis}

All continuous variables were expressed as mean \pm SEM. After checking for skewness and kurtosis, insulin, HOMA-IR, total cholesterol, MMP-9 and total PAI-1 were normalized using logarithmic transformation. Homogeneity of variances was estimated using the Levene test. Comparisons between obese and normal-BMI children variables were assessed using the Student's t-test for unpaired samples using SPSS (version 15.0.1. Chicago, IL). Genotype and allele frequencies were calculated for cases and controls. The genotypic relative risk was assessed by comparing the obese and normal-BMI group, calculating the odds ratio (OR) and 95\% confidence interval (CI), using logistic regression analysis under an additive model adjusted by sex and age with a Bonferroni correction using the SNPassoc package from R software [18]. Logistic or linear regressions analysis in the entire population was performed under an additive model to estimate the associations of each SNP with phenotypic parameters related to obesity as well as biomarkers of CVD risk and inflammation, adjusted by sex and age using the PLINK software. Haplotype analysis involved the use of Haploview version 4.2 software and haplo.stats package from $\mathrm{R}$ software. Logistic regression analyses was performed to assess the association of the studied haplotypes with obesity; the model generated computes a $x^{2}$ to estimate the $p$ value that test the fit of the model. This $\mathrm{p}$ explains the variation in the phenotype compared to a model with no effect. Significance was considered at the level of $\mathrm{p}<0.05$.

\section{Results}

\section{General characteristics of the population}

Table 1 shows the anthropometric, clinical and metabolic characteristics of obese and normal-BMI subjects. Weight, height, BMI, BMI z-score and WC were significantly higher in the obese compared with the normalBMI children. Systolic and diastolic BP, as well as plasma triacylglycerols, apolipoprotein B (ApoB), insulin, and HOMA-IR, were higher in obese children, whereas plasmatic total cholesterol, high-density lipoproteincholesterol (HDL-c) and apolipoprotein A1 (ApoA1) were lower in obese children. Fasting plasma glucose and lowdensity lipoprotein-cholesterol (LDL-c) concentrations did not show differences between groups. Plasmatic resistin and leptin concentrations were significantly higher in obese than in normal-BMI subjects, by contrast decreased levels of adiponectin were found in obese children. Inflammatory and CVD risk biomarkers were different between groups. CRP, IL-6, IL- 8 and TNF- $\alpha$ were significantly higher in the obese group compared with those in the normal-BMI group. Likewise, plasmatic sICAM-1, sEselectin, MPO and active and total PAI-1 were higher in the obese group, whereas MMP-9 levels did not show difference between groups.

\section{Association of SNPs with obesity}

Five SNPs of the 52 included in the analysis were deviated from Hardy-Weinberg equilibrium in the normalBMI group $(\mathrm{p}<0.05)$. Those SNPs were excluded from the association analysis. Of the 47 SNPs, five were associated with obesity in children, adjusted by age and sex under an additive model after Bonferroni correction; the rs9935401, rs9939609, rs9928094 and rs9930333 located in the intron 1 were positively associated with obesity, whereas the rs8061518 in the intron 3 was negatively associated (Table 2).

\section{Association of SNPs with obesity-related traits}

Table 3 shows the association of the rs 9939609 with obesity relate-traits. MMP-9 was the only biomarker associated with the former SNP after adjustment for sex, age and BMI [ $\beta=0.03 \mu \mathrm{g} / \mathrm{l} ; 95 \%$ CI: $(0.0004,0.0690] \quad(\mathrm{p}=0.048)$. 
Table 1 Anthropometric, clinical and biochemical parameters of the children

\begin{tabular}{cccc}
\hline & Normal-weight & Obese & P \\
\hline $\mathrm{n}$ & 242 & 292 &
\end{tabular}

Anthropometric factors

\begin{tabular}{|c|c|c|c|}
\hline $\operatorname{Sex}(M / F)$ & $135 / 107$ & $149 / 143$ & 0.273 \\
\hline Age (y) & $9.73 \pm 0.2$ & $9.43 \pm 0.2$ & 0.172 \\
\hline Weight (kg) & $32.9 \pm 0.7$ & $55.9 \pm 1.0$ & $<0.001$ \\
\hline Height (m) & $1.37 \pm 0.01$ & $1.41 \pm 0.01$ & 0.001 \\
\hline BMI $\left(\mathrm{kg} / \mathrm{m}^{2}\right)$ & $17.14 \pm 0.13$ & $27.56 \pm 0.24$ & $<0.001$ \\
\hline BMI z-score & $-0.17 \pm 0.04$ & $3.49 \pm 0.08$ & $<0.001$ \\
\hline Waist circumference (cm) & $60.2 \pm 0.5$ & $84.0 \pm 0.9$ & $<0.001$ \\
\hline \multicolumn{4}{|c|}{ Clinical and metabolic biomarkers } \\
\hline Systolic BP (mm Hg) & $97 \pm 1$ & $111 \pm 1$ & $<0.001$ \\
\hline Diastolic BP (mm Hg) & $60 \pm 1$ & $69 \pm 1$ & $<0.001$ \\
\hline Glucose (mg/dl) & $84 \pm 0$ & $85 \pm 1$ & 0.772 \\
\hline Insulin (mU/l) & $5.95 \pm 0.23$ & $11.46 \pm 0.51$ & $<0.001$ \\
\hline HOMA-IR & $1.26 \pm 0.05$ & $2.43 \pm 0.12$ & $<0.001$ \\
\hline Triacylglycerols (mg/dl) & $55 \pm 1$ & $75 \pm 2$ & $<0.001$ \\
\hline ApoAl (mg/dl) & $150 \pm 2$ & $132 \pm 1$ & $<0.001$ \\
\hline ApoB (mg/dl) & $67 \pm 1$ & $71 \pm 1$ & 0.008 \\
\hline Cholesterol (mg/dl) & $171 \pm 2$ & $165 \pm 2$ & 0.012 \\
\hline $\mathrm{HDL}-\mathrm{c}(\mathrm{mg} / \mathrm{dl})$ & $64 \pm 1$ & $51 \pm 1$ & $<0.001$ \\
\hline LDL-c (mg/dl) & $94 \pm 2$ & $97 \pm 1$ & 0.144 \\
\hline Adiponectin (mg/l) & $28.28 \pm 0.77$ & $22.57 \pm 0.66$ & $<0.001$ \\
\hline Resistin $(\mu \mathrm{g} / \mathrm{l})$ & $9.65 \pm 0.33$ & $11.71 \pm 0.34$ & $<0.001$ \\
\hline Leptin $(\mu \mathrm{g} / \mathrm{l})$ & $4.30 \pm 0.26$ & $22.91 \pm 0.86$ & $<0.001$ \\
\hline \multicolumn{4}{|l|}{ Inflammatory biomarkers } \\
\hline C-reactive protein (mg/l) & $0.48 \pm 0.07$ & $1.99 \pm 0.16$ & $<0.001$ \\
\hline Interleukin 6 (ng/l) & $4.50 \pm 0.53$ & $6.95 \pm 0.75$ & 0.012 \\
\hline Interleukin 8 (ng/l) & $1.57 \pm 0.11$ & $2.16 \pm 0.15$ & 0.003 \\
\hline TNF-a (ng/l) & $3.06 \pm 0.11$ & $4.01 \pm 0.13$ & $<0.001$ \\
\hline \multicolumn{4}{|c|}{ Cardiovascular disease risk biomarkers } \\
\hline MMP-9 $(\mu \mathrm{g} / \mathrm{l})$ & $79.99 \pm 3.15$ & $88.42 \pm 3.90$ & 0.636 \\
\hline MPO ( $\mu \mathrm{g} / \mathrm{l})$ & $13.10 \pm 1.16$ & $21.51 \pm 1.70$ & $<0.001$ \\
\hline sE selectin $(\mu \mathrm{g} / \mathrm{l})$ & $22.93 \pm 0.76$ & $31.26 \pm 1.05$ & $<0.001$ \\
\hline sICAM-1 (mg/l) & $0.153 \pm 0.004$ & $0.175 \pm 0.005$ & $<0.001$ \\
\hline Active PAl-1 ( $\mu \mathrm{g} / \mathrm{l})$ & $5.16 \pm 0.27$ & $11.96 \pm 0.57$ & $<0.001$ \\
\hline Total PAI-1 ( $\mu \mathrm{g} / \mathrm{l})$ & $18.95 \pm 0.85$ & $27.23 \pm 1.01$ & $<0.001$ \\
\hline
\end{tabular}

M: male; F: female; BMI: body mass index; BP: blood pressure; HOMA-IR: homeostasis model assessment for insulin resistance; Apo: apolipoprotein; HDL-c: high-density lipoprotein cholesterol; LDL-c: low-density lipoprotein cholesterol; TNF-a: tumor necrosis factor alpha; MMP-9: metalloproteinase-9; MPO: myeloperoxidase; sICAM-1: soluble intracellular adhesion molecule-1, PAl-1: plasminogen activator inhibitor.
The analyses of the other SNPs positive associated with obesity are included in the Additional file 1: Tables S2-S4.

Table 4 shows the association of the rs 8061518 with obesity relate-traits. Genotype with the minor allele of this variant was negatively associated with leptin after adjustment for age, sex and BMI $[\beta=-1.38 \mu \mathrm{g} / \mathrm{l}(95 \%$ CI: $-2.70,-0.06)](\mathrm{p}=0.041)$.

\section{Linkage disequilibrium and haplotype block analysis}

Figure 1 shows the LD of two well-defined haplo-blocks. In this figure, we can observe the LD between the 5 SNPs significantly associated with obesity. rs9928094, rs9930333, rs9935401 and rs9939609 are in LD between each other, while rs8061518 is in LD with no one of the above. Table 5 shows the total population haplotype blocks frequency as well as cases and controls separately. The haplo-block 1 involves the SNPs rs9928094, rs9930333, rs10852521, rs11075986, rs9935401 and rs9939609. The overall $p$ value of $\chi^{2}$ for association with obesity for this haplo-block was $8.72 \times 10^{-4}$. The risk for obesity associated with the haplotype GGCCAA (which include the risk allele of rs9939609A and the risk alleles of the rs9928094-G, rs9930333-G and rs9935401-A all of them in LD with the former) was $1.26(95 \% \mathrm{CI}: 1.64-2.14)\left(\mathrm{p}=5.11 \times 10^{-5}\right)$. The haplo-block 2 involves the SNPs rs3826169, rs8061518, rs17818902 and rs7190053. The overall $\mathrm{p}$ value of $x^{2}$ for association with obesity for this haplo-block was 0.026 . The risk for obesity associated with the haplotype TGTC (the only one that include the rs8061518-G allele, that showed a negative association with obesity) was 0.65 (95\% CI: $0.47-0.92)(\mathrm{p}=0.002)$.

\section{Discussion}

In the present study, where we studied 52 FTO variants spanning the whole gene, we validate the positive association of rs9939609 with obesity in Spanish children. We found three other SNPs (rs9935401, rs9928094, and 9930333), also localized in the first intron of the gene positively associated with obesity. Additionally, we observed for the first time, the association of rs8061518, localized in the third intron of the gene, with a reduced risk of obesity and low plasma leptin concentrations.

Our results are in concordance with many studies, which have shown the association of the rs9939609 with adulthood and childhood obesity [3,19], as well as other SNPs in LD with it, such as the rs9935401, rs9928094 and rs9930333 [16,20,21]. Several studies among European descent population have demonstrated the association of variant rs9939609 with anthropometric parameters, such as BMI, weight, WC [22] and skin folds [23]. Interestingly, the association with BMI found in carriers of haplotype GGCCAA (which include the risk alleles of the SNPs positively associated with obesity) could be useful for the early identification of inherited susceptibility to weight-gain 
Table 2 Genotypic distribution of the FTO analysed polymorphisms and its association with obesity

\begin{tabular}{|c|c|c|c|c|c|c|c|c|c|c|c|c|c|}
\hline & & & Case & & & ontro & & & Mino & Allele & & & \\
\hline Polymorphism & Allele 1 /allele 2 & 11 & 12 & 22 & 11 & 12 & 22 & Minor allele & Case & Control & OR $(95 \% \mathrm{Cl})$ & $\mathbf{P}$ & P corr* \\
\hline rs 12445162 & $\mathrm{G} / \mathrm{A}$ & 253 & 39 & 0 & 203 & 37 & 2 & A & 0.066 & 0.085 & $0.63(0.35-1.11)$ & 0.108 & 1 \\
\hline rs11075986 & $C / G$ & 249 & 42 & 1 & 200 & 38 & 4 & G & 0.075 & 0.097 & $1.07(0.80-1.44)$ & 0.630 & 1 \\
\hline rs7203521 & $A / G$ & 110 & 143 & 39 & 90 & 116 & 36 & G & 0.381 & 0.388 & $0.93(0.68-1.28)$ & 0.667 & 1 \\
\hline rs1861868 & $A / G$ & 85 & 155 & 52 & 63 & 118 & 60 & G & 0.453 & 0.494 & $0.78(0.58-1.06)$ & 0.108 & 1 \\
\hline rs11643744 & $\mathrm{A} / \mathrm{G}$ & 157 & 117 & 18 & 123 & 104 & 14 & G & 0.268 & 0.278 & $0.86(0.61-1.22)$ & 0.404 & 1 \\
\hline rs9928094 & $A / G$ & 67 & 149 & 75 & 88 & 114 & 40 & G & 0.511 & 0.398 & $1.66(1.22-2.24)$ & 0.0009 & 0.018 \\
\hline rs9930333 & T/G & 67 & 150 & 74 & 87 & 112 & 41 & G & 0.511 & 0.398 & $1.64(1.21-2.22)$ & 0.001 & 0.023 \\
\hline rs 10852521 & $\mathrm{C} / \mathrm{T}$ & 104 & 139 & 49 & 64 & 116 & 61 & $\mathrm{~T}$ & 0.410 & 0.496 & $0.67(0.49-0.90)$ & 0.008 & 0.327 \\
\hline rs7205986 & $\mathrm{A} / \mathrm{G}$ & 68 & 151 & 72 & 70 & 111 & 61 & G & 0.505 & 0.483 & $0.78(0.46-1.34)$ & 0.369 & 1 \\
\hline rs9935401 & $G / A$ & 72 & 150 & 69 & 95 & 111 & 33 & A & 0.419 & 0.370 & $1.76(1.29-2.41)$ & 0.0003 & 0.004 \\
\hline rs9939609 & $T / A$ & 72 & 149 & 69 & 90 & 118 & 33 & A & 0.491 & 0.377 & $1.73(1.26-2.37)$ & 0.0006 & 0.009 \\
\hline rs3826169 & $\mathrm{T} / \mathrm{C}$ & 170 & 102 & 20 & 151 & 81 & 10 & C & 0.245 & 0.210 & $1.32(0.92-1.89)$ & 0.132 & 1 \\
\hline rs8061518 & $A / G$ & 159 & 107 & 22 & 95 & 108 & 32 & G & 0.262 & 0.365 & $0.56(0.40-0.78)$ & 0.0006 & 0.025 \\
\hline rs17818902 & $T / G$ & 162 & 107 & 22 & 145 & 84 & 13 & G & 0.261 & 0.227 & $1.19(0.84-1.67)$ & 0.329 & 1 \\
\hline rs7190053 & $\mathrm{C} / \mathrm{T}$ & 190 & 87 & 13 & 165 & 67 & 7 & $\mathrm{~T}$ & 0.196 & 0.172 & $1.21(0.82-1.78)$ & 0.338 & 1 \\
\hline rs2111114 & $A / G$ & 175 & 107 & 10 & 151 & 81 & 9 & G & 0.219 & 0.203 & $1.19(0.82-1.74)$ & 0.362 & 1 \\
\hline rs8044353 & $\mathrm{G} / \mathrm{A}$ & 259 & 31 & 1 & 219 & 23 & 0 & A & 0.056 & 0.047 & $1.21(0.61-2.39)$ & 0.589 & 1 \\
\hline rs 10521303 & $C / A$ & 91 & 151 & 48 & 64 & 133 & 42 & A & 0.400 & 0.436 & $0.84(0.62-1.15)$ & 0.284 & 1 \\
\hline rs1558756 & $\mathrm{C} / \mathrm{T}$ & 86 & 152 & 54 & 69 & 132 & 41 & $\mathrm{~T}$ & 0.441 & 0.443 & $1.14(0.83-1.57)$ & 0.421 & 1 \\
\hline rs 16952623 & $\mathrm{~T} / \mathrm{C}$ & 215 & 72 & 3 & 181 & 58 & 2 & C & 0.134 & 0.128 & $0.98(0.62-1.56)$ & 0.930 & 1 \\
\hline rs 16952624 & $\mathrm{C} / \mathrm{T}$ & 289 & 1 & 0 & 242 & 0 & 0 & $\mathrm{~T}$ & 0.002 & 0.000 & & & 1 \\
\hline rs2111113 & $\mathrm{G} / \mathrm{C}$ & 247 & 45 & 0 & 208 & 30 & 2 & C & 0.079 & 0.068 & $1.00(0.56-1.78)$ & 0.996 & 1 \\
\hline rs 10852525 & $\mathrm{G} / \mathrm{A}$ & 229 & 59 & 1 & 194 & 41 & 5 & A & 0.105 & 0.105 & $0.89(0.55-1.45)$ & 0.641 & 1 \\
\hline rs7194336 & $\mathrm{G} / \mathrm{T}$ & 98 & 144 & 50 & 63 & 131 & 48 & $\mathrm{~T}$ & 0.418 & 0.470 & $0.74(0.55-1.01)$ & 0.053 & 1 \\
\hline rs7203181 & $C / A$ & 142 & 111 & 39 & 101 & 109 & 32 & A & 0.329 & 0.356 & $0.88(0.65-1.19)$ & 0.412 & 1 \\
\hline rs6499656 & $\mathrm{G} / \mathrm{C}$ & 218 & 67 & 7 & 189 & 48 & 5 & C & 0.140 & 0.121 & $1.30(0.84-2.03)$ & 0.237 & 1 \\
\hline rs7191513 & $\mathrm{G} / \mathrm{A}$ & 98 & 145 & 49 & 82 & 110 & 50 & A & 0.418 & 0.441 & $0.96(0.72-1.30)$ & 0.805 & 1 \\
\hline rs7194907 & $\mathrm{T} / \mathrm{C}$ & 81 & 153 & 57 & 65 & 105 & 67 & C & 0.458 & 0.509 & $0.87(0.64-1.18)$ & 0.370 & 1 \\
\hline rs8056299 & $\mathrm{A} / \mathrm{G}$ & 104 & 140 & 47 & 77 & 116 & 49 & G & 0.402 & 0.441 & $0.84(0.62-1.14)$ & 0.255 & 1 \\
\hline rs 17225435 & $A / G$ & 225 & 61 & 6 & 194 & 46 & 2 & G & 0.122 & 0.104 & $1.51(0.93-2.46)$ & 0.093 & 1 \\
\hline rs8049235 & $\mathrm{G} / \mathrm{A}$ & 100 & 156 & 34 & 91 & 108 & 43 & A & 0.383 & 0.396 & $1.01(0.74-1.59)$ & 0.930 & 1 \\
\hline rs7199716 & $\mathrm{C} / \mathrm{T}$ & 101 & 146 & 44 & 91 & 116 & 35 & $\mathrm{~T}$ & 0.402 & 0.384 & $0.91(0.67-1.24)$ & 0.547 & 1 \\
\hline rs13334214 & $C / T$ & 178 & 102 & 6 & 155 & 71 & 13 & $\mathrm{~T}$ & 0.198 & 0.204 & $1.07(0.74-1.57)$ & 0.710 & 1 \\
\hline rs7194243 & $\mathrm{C} / \mathrm{T}$ & 180 & 93 & 19 & 147 & 87 & 8 & $\mathrm{~T}$ & 0.227 & 0.214 & $0.82(0.57-1.18)$ & 0.295 & 1 \\
\hline rs1136002 & $\mathrm{T} / \mathrm{C}$ & 135 & 130 & 27 & 109 & 111 & 22 & C & 0.316 & 0.320 & $0.90(0.66-1.24)$ & 0.529 & 1 \\
\hline rs4784351 & $\mathrm{A} / \mathrm{G}$ & 154 & 113 & 20 & 122 & 96 & 16 & G & 0.268 & 0.275 & $0.92(0.65-1.28)$ & 0.612 & 1 \\
\hline rs2540781 & $\mathrm{C} / \mathrm{A}$ & 225 & 54 & 13 & 190 & 47 & 5 & A & 0.136 & 0.119 & $0.87(0.57-1.32)$ & 0.507 & 1 \\
\hline rs8049933 & $\mathrm{C} / \mathrm{T}$ & 231 & 52 & 7 & 195 & 41 & 4 & $\mathrm{~T}$ & 0.115 & 0.103 & $0.83(0.52-1.32)$ & 0.425 & 1 \\
\hline rs1558687 & $\mathrm{C} / \mathrm{T}$ & 169 & 107 & 15 & 134 & 92 & 14 & $\mathrm{~T}$ & 0.233 & 0.250 & $0.94(0.67-1.33)$ & 0.738 & 1 \\
\hline rs2075202 & $T / G$ & 277 & 14 & 1 & 231 & 11 & 0 & G & 0.026 & 0.023 & $0.69(0.25-1.88)$ & 0.469 & 1 \\
\hline rs7200579 & $C / G$ & 257 & 33 & 2 & 216 & 25 & 1 & G & 0.065 & 0.055 & $0.91(0.50-1.68)$ & 0.772 & 1 \\
\hline rs697771 & $C / T$ & 102 & 137 & 53 & 72 & 127 & 43 & $\mathrm{~T}$ & 0.413 & 0.443 & $1.00(0.74-1.36)$ & 0.985 & 1 \\
\hline rs12596638 & $\mathrm{G} / \mathrm{A}$ & 221 & 66 & 4 & 176 & 61 & 5 & A & 0.126 & 0.150 & $0.99(0.64-1.53)$ & 0.979 & 1 \\
\hline
\end{tabular}


Table 2 Genotypic distribution of the FTO analysed polymorphisms and its association with obesity (Continued)

\begin{tabular}{ccccccccccccccc}
\hline rs1008400 & $C / T$ & 81 & 148 & 61 & 67 & 132 & 43 & $T$ & 0.469 & 0.451 & $0.97(0.71-1.32)$ & 0.860 & 1 \\
rs12932373 & $C / T$ & 214 & 76 & 2 & 175 & 61 & 3 & $T$ & 0.136 & 0.139 & $0.77(0.49-1.21)$ & 0.261 & 1 \\
rs2689248 & $C / A$ & 79 & 153 & 59 & 64 & 113 & 63 & $\mathrm{~A}$ & 0.463 & 0.492 & $0.79(0.59-1.07)$ & 0.124 & 1 \\
rs17833492 & $\mathrm{C} / \mathrm{A}$ & 140 & 127 & 24 & 106 & 112 & 23 & $\mathrm{~A}$ & 0.296 & 0.322 & $0.88(0.63-1.22)$ & 0.437 & 1 \\
\hline
\end{tabular}

$\mathrm{Cl}$ : confidence interval; OR: odds ratio. The OR was adjusted for age and sex under the additive model. * $\mathrm{P}$ values after Bonferroni correction. SNPs are ordered according to their genomic position.

Table 3 Association of rs9939609 with anthropometric, clinical, inflammation and CVD risk biomarkers in children

\begin{tabular}{lllllll}
\hline Biomarkers & TT & AT & AA & $\boldsymbol{\beta}(\mathbf{9 5} \% \mathrm{Cl})$ & $\mathbf{P}$ & $\mathbf{P}^{\mathbf{a}}$ \\
\hline $\mathrm{n}$ & 162 & 267 & 102 & & &
\end{tabular}

Anthropometric factors

\begin{tabular}{|c|c|c|c|c|c|c|}
\hline Height (m) & $1.38 \pm 0.01$ & $1.39 \pm 0.01$ & $1.40 \pm 0.02$ & $-0.003(-0.01,0.00)$ & 0.148 & 0.594 \\
\hline Weight (kg) & $42.3 \pm 1.4$ & $45.6 \pm 1.1$ & $50.1 \pm 2.2$ & $3.31(1.52,5.10)$ & 0.001 & 0.397 \\
\hline $\mathrm{BMI}\left(\mathrm{kg} / \mathrm{m}^{2}\right)$ & $21.69 \pm 0.48$ & $22.89 \pm 0.36$ & $24.51 \pm 0.64$ & $1.32(0.59,2.05)$ & 0.001 & - \\
\hline BMI z-score & $1.48 \pm 0.16$ & $1.86 \pm 0.13$ & $2.32 \pm 0.22$ & $0.45(0.19,0.71)$ & 0.001 & - \\
\hline Waist circumference $(\mathrm{cm})$ & $70.58 \pm 1.39$ & $72.85 \pm 0.99$ & $77.46 \pm 1.71$ & $3.35(0.61,6.10)$ & 0.017 & 0.487 \\
\hline \multicolumn{7}{|c|}{ Clinical and metabolic biomarkers } \\
\hline Systolic BP (mm Hg) & $104 \pm 1$ & $104 \pm 1$ & $107 \pm 1$ & $1.14(-0.71,2.99)$ & 0.227 & 0.466 \\
\hline Diastolic BP (mm Hg) & $63 \pm 1$ & $65 \pm 1$ & $66 \pm 1$ & $1.24(-0.26,2.74)$ & 0.106 & 0.888 \\
\hline Glucose (mg/dl) & $85 \pm 1$ & $84 \pm 0$ & $84 \pm 1$ & $-0.47(-1.34,0.39)$ & 0.286 & 0.143 \\
\hline Insulin (mU/l) & $8.82 \pm 0.64$ & $8.73 \pm 0.43$ & $9.78 \pm 0.71$ & $0.02(-0.01,0.05)$ & 0.253 & 0.225 \\
\hline HOMA-IR & $1.89 \pm 0.15$ & $1.85 \pm 0.10$ & $2.07 \pm 0.16$ & $0.02(-0.02,0.05)$ & 0.345 & 0.181 \\
\hline Triacylglycerols (mg/dl) & $67 \pm 3$ & $64 \pm 2$ & $68 \pm 4$ & $0.07(-3.90,4.03)$ & 0.974 & 0.216 \\
\hline ApoAl (mg/dl) & $142 \pm 2$ & $141 \pm 2$ & $135 \pm 3$ & $-3.01(-6.36,0.34)$ & 0.079 & 0.457 \\
\hline Cholesterol (mg/dl) & $169 \pm 3$ & $166 \pm 2$ & $170 \pm 2$ & $0.002(-0.01,0.01)$ & 0.721 & 0.540 \\
\hline $\mathrm{HDL}-\mathrm{c}(\mathrm{mg} / \mathrm{dl})$ & $57 \pm 1$ & $57 \pm 1$ & $55 \pm 1$ & $-0.94(-2.76,0.89)$ & 0.316 & 0.509 \\
\hline Adiponectin (mg/l) & $25.89 \pm 0.95$ & $25.13 \pm 0.74$ & $24.16 \pm 1.10$ & $-0.72(-2.18,0.73)$ & 0.331 & 0.934 \\
\hline Resistin ( $\mu \mathrm{g} / \mathrm{l})$ & $10.33 \pm 0.44$ & $10.81 \pm 0.35$ & $11.43 \pm 0.55$ & $0.55(-0.15,1.25)$ & 0.125 & 0.384 \\
\hline Leptin $(\mu \mathrm{g} / \mathrm{l})$ & $13.57 \pm 1.23$ & $14.37 \pm 0.83$ & $16.29 \pm 1.52$ & $1.38(-0.43,3.18)$ & 0.135 & 0.149 \\
\hline \multicolumn{7}{|l|}{ Inflammatory biomarkers } \\
\hline C-reactive protein (mg/l) & $2.08 \pm 0.29$ & $2.21 \pm 0.23$ & $2.77 \pm 0.53$ & $0.37(-0.14,0.88)$ & 0.153 & 0.743 \\
\hline IL-6 (ng/l) & $5.61 \pm 0.90$ & $6.00 \pm 0.69$ & $6.19 \pm 1.05$ & $0.42(-0.96,1.80)$ & 0.550 & 0.759 \\
\hline IL-8 (ng/l) & $1.86 \pm 0.16$ & $1.95 \pm 0.15$ & $1.83 \pm 0.21$ & $0.03(-0.25,0.30)$ & 0.853 & 0.783 \\
\hline TNF-a (ng/l) & $3.53 \pm 0.16$ & $3.66 \pm 0.13$ & $3.33 \pm 0.20$ & $-0.02(-0.27,0.23)$ & 0.875 & 0.308 \\
\hline \multicolumn{7}{|c|}{ Cardiovascular disease risk biomarkers } \\
\hline MMP-9 $(\mu \mathrm{g} / \mathrm{l})$ & $80.39 \pm 4.35$ & $83.62 \pm 3.70$ & $92.82 \pm 6.05$ & $0.03(0.00,0.07)$ & 0.049 & 0.048 \\
\hline MPO ( $\mu \mathrm{g} / \mathrm{l})$ & $17.56 \pm 2.06$ & $17.84 \pm 1.61$ & $17.65 \pm 1.88$ & $0.29(-2.80,3.38)$ & 0.853 & 0.636 \\
\hline sE-Selectin $(\mu \mathrm{g} / \mathrm{l})$ & $26.34 \pm 1.14$ & $28.22 \pm 1.05$ & $26.64 \pm 1.40$ & $0.66(-1.25,2.56)$ & 0.500 & 0.711 \\
\hline sICAM-1 (mg/l) & $0.162 \pm 0.005$ & $0.168 \pm 0.004$ & $0.158 \pm 0.006$ & $0.001(-0.01,0.01)$ & 0.997 & 0.645 \\
\hline Active PAl-1 $(\mu \mathrm{g} / \mathrm{l})$ & $8.35 \pm 0.58$ & $8.61 \pm 0.52$ & $10.35 \pm 0.97$ & $0.85(-0.19,1.89)$ & 0.109 & .946 \\
\hline Total PAl-1 $(\mu \mathrm{g} / \mathrm{l})$ & $23.22 \pm 1.34$ & $22.21 \pm 0.92$ & $26.84 \pm 2.09$ & $0.02(-0.02,0.06)$ & 0.359 & 0.994 \\
\hline
\end{tabular}

$\mathrm{Cl}$ : Confidence interval; BMI: body mass index; BP: blood pressure; HOMA-IR: homeostasis model assessment for insulin resistance; HDL-c: high-density lipoprotein cholesterol; IL: interleukin; TNF-a: tumour necrosis factor alpha; MMP-9: matrix metalloproteinase-9; MPO: myeloperoxidase; sICAM-1: soluble intracellular adhesion molecule-1, PAl-1: plasminogen activator inhibitor. $\beta$ Coefficients represent the change in absolute traits values of each additional risk allele. General linear models were used to examine associations, $\mathrm{P}$ adjusted by age and sex, $\mathrm{P}^{\mathrm{a}}$ adjusted by age, sex, and BMI. 
Table 4 Association of rs8061518 with anthropometric, clinical, inflammation and CVD risk biomarkers in children

\begin{tabular}{lccccccc}
\hline Biomarkers & AA & AG & GG & $\beta(95 \%$ Cl) & P & $P^{a}$ \\
\hline $\mathrm{N}$ & 254 & 215 & 54 & & &
\end{tabular}

Anthropometric factors

\begin{tabular}{|c|c|c|c|c|c|c|}
\hline Height (m) & $1.39 \pm 0.01$ & $1.40 \pm 0.01$ & $1.39 \pm 0.02$ & $-0.001(-0.01,0.00)$ & 0.613 & 0.107 \\
\hline Weight (kg) & $46.8 \pm 1.2$ & $44.9 \pm 1.3$ & $42.6 \pm 2.4$ & $-2.44(-4.35,-0.54)$ & 0.012 & 0.130 \\
\hline $\mathrm{BMI}\left(\mathrm{kg} / \mathrm{m}^{2}\right)$ & $23.70 \pm 0.38$ & $22.33 \pm 0.42$ & $21.48 \pm 0.81$ & $-1.27(-2.05,-0.50)$ & 0.001 & - \\
\hline BMI z-score & $2.16 \pm 0.14$ & $1.61 \pm 0.14$ & $1.36 \pm 0.30$ & $-0.45(-0.72,-0.17)$ & 0.001 & - \\
\hline Waist circumference $(\mathrm{cm})$ & $74.74 \pm 1.07$ & $71.99 \pm 1.18$ & $71.10 \pm 2.25$ & $-1.70(-4.63,1.22)$ & 0.254 & 0.534 \\
\hline \multicolumn{7}{|c|}{ Clinical and metabolic biomarkers } \\
\hline Systolic BP (mm Hg) & $106 \pm 1$ & $103 \pm 1$ & $104 \pm 2$ & $-1.74(-3.69,0.21)$ & 0.080 & 0.758 \\
\hline Diastolic BP (mm Hg) & $66 \pm 1$ & $64 \pm 1$ & $61 \pm 1$ & $-2.23(-3.80,-0.65)$ & 0.006 & 0.083 \\
\hline Glucose (mg/dl) & $85 \pm 0$ & $84 \pm 0$ & $85 \pm 1$ & $-0.11(-1.02,0.80)$ & 0.816 & 0.836 \\
\hline Insulin (mU/l) & $9.52 \pm 0.48$ & $8.56 \pm 0.48$ & $8.17 \pm 1.13$ & $-0.05(-0.09,-0.02)$ & 0.003 & 0.153 \\
\hline HOMA-IR & $2.02 \pm 0.10$ & $1.80 \pm 0.11$ & $1.79 \pm 0.30$ & $-0.05(-0.09,-0.02)$ & 0.003 & 0.153 \\
\hline Triacylglycerols (mg/dl) & $68 \pm 2$ & $63 \pm 2$ & $66 \pm 6$ & $-2.33(-6.52,1.86)$ & 0.276 & 0.995 \\
\hline ApoAl (mg/dl) & $141 \pm 2$ & $137 \pm 2$ & $145 \pm 4$ & $0.15(-3.40,3.71)$ & 0.932 & 0.330 \\
\hline Cholesterol (mg/dl) & $166 \pm 2$ & $168 \pm 2$ & $172 \pm 4$ & $0.01(-0.004,0.02)$ & 0.223 & 0.287 \\
\hline $\mathrm{HDL}-\mathrm{c}(\mathrm{mg} / \mathrm{dl})$ & $57 \pm 1$ & $56 \pm 1$ & $58 \pm 2$ & $0.07(-1.87,2.01)$ & 0.945 & 0.107 \\
\hline Adiponectin (mg/l) & $25.46 \pm 0.77$ & $23.92 \pm 0.73$ & $27.90 \pm 1.81$ & $0.38(-1.15,1.91)$ & 0.628 & 0.741 \\
\hline Resistin ( $\mu \mathrm{g} / \mathrm{l})$ & $11.34 \pm 0.40$ & $10.30 \pm 0.33$ & $10.37 \pm 0.72$ & $-0.68(-1.42,0.06)$ & 0.074 & 0.227 \\
\hline Leptin $(\mu \mathrm{g} / \mathrm{l})$ & $16.98 \pm 1.02$ & $12.57 \pm 0.85$ & $11.21 \pm 1.70$ & $-3.55(-5.43,-1.66)$ & $<0.001$ & 0.041 \\
\hline \multicolumn{7}{|l|}{ Inflammatory biomarkers } \\
\hline C-reactive protein (mg/l) & $2.50 \pm 0.26$ & $2.26 \pm 0.31$ & $1.70 \pm 0.30$ & $-0.34(-0.89,0.20)$ & 0.217 & 0.784 \\
\hline IL-6 (ng/l) & $5.62 \pm 0.64$ & $6.56 \pm 0.90$ & $4.71 \pm 0.91$ & $0.09(-1.36,1.53)$ & 0.905 & 0.705 \\
\hline IL-8 (ng/l) & $1.94 \pm 0.14$ & $1.98 \pm 0.17$ & $1.57 \pm 0.19$ & $-0.10(-0.39,0.19)$ & 0.495 & 0.804 \\
\hline TNFa (ng/l) & $3.62 \pm 0.14$ & $3.51 \pm 0.14$ & $3.60 \pm 0.27$ & $-0.05(-0.32,0.22)$ & 0.711 & 0.696 \\
\hline \multicolumn{7}{|c|}{ Cardiovascular disease risk biomarkers } \\
\hline MMP-9 ( $\mu \mathrm{g} / \mathrm{l})$ & $82.62 \pm 3.42$ & $86.21 \pm 4.14$ & $84.12 \pm 8.05$ & $0.01(-0.03,0.04)$ & 0.764 & 0.754 \\
\hline MPO ( $\mu \mathrm{g} / \mathrm{l})$ & $19.19 \pm 1.68$ & $16.65 \pm 1.44$ & $16.55 \pm 4.26$ & $-1.63(-4.94,1.68)$ & 0.336 & 0.712 \\
\hline sE-Selectin $(\mu \mathrm{g} / \mathrm{l})$ & $28.19 \pm 0.98$ & $26.13 \pm 1.08$ & $26.61 \pm 2.34$ & $-1.09(-3.13,0.95)$ & 0.296 & 0.924 \\
\hline sICAM-1 (mg/l) & $0.165 \pm 0.004$ & $0.168 \pm 0.005$ & $0.155 \pm 0.007$ & $-0.002(-0.01,0.01)$ & 0.605 & 0.923 \\
\hline Active PAl-1 ( $\mu \mathrm{g} / \mathrm{l})$ & $9.81 \pm 0.62$ & $8.45 \pm 0.49$ & $6.16 \pm 0.63$ & $-1.71(-2.80,-0.62)$ & 0.002 & 0.067 \\
\hline Total PAl-1 $(\mu \mathrm{g} / \mathrm{l})$ & $24.74 \pm 1.11$ & $22.56 \pm 1.16$ & $20.66 \pm 1.85$ & $-0.04(-0.08,0.003)$ & 0.073 & 0.350 \\
\hline
\end{tabular}

Cl: Confidence Interval; BMI: body mass index; BP: blood pressure; HOMA-IR: homeostasis model assessment for insulin resistance; HDL-c: High-density lipoproteincholesterol; IL: interleukin; TNFa: tumour necrosis factor alpha; MMP-9: matrix metalloproteinase-9; MPO: myeloperoxidase; sICAM-1: soluble intracellular adhesion molecule-1; PAl-1: plasminogen activator inhibitor-1. $\beta$ coefficients represent the change in absolute traits values of each additional risk allele. General linear models were used to examine associations, $\mathrm{P}$ adjusted by age and sex, $\mathrm{P}^{\mathrm{a}}$ adjusted by age, sex, and BMI.

beginning during the childhood, with a higher sensitivity than considering the effect of a single marker.

Among the large number of phenotypes measured in this study, we did not find any significant association with FTO variants located in the first intron after additional adjustment for BMI, except in the case of MMP-9, a wellknown biomarker of CVD risk [24], which has shown an association not only with rs9939609 but also with rs9928094 and rs9930333. The association of rs9939609 with CVD markers are opposed in the scientific literature.
Whereas some have shown significant association with low HDL-c [25,26] and high triacylglycerols [25], others $[27,28]$ have not found any association. Additionally, there are few studies that link CVD and FTO gene [29,30], and to our knowledge, there are no studies that have included CVD risk biomarkers as MPO, sE-selectin, sICAM-1, PAI1 and MMP-9 as we did here. Some other studies have shown the association of rs9939609 with inflammatory biomarkers as in the case of Timpson et al. [31] and Fisher et al. [32]; both found a positive association with CRP. 


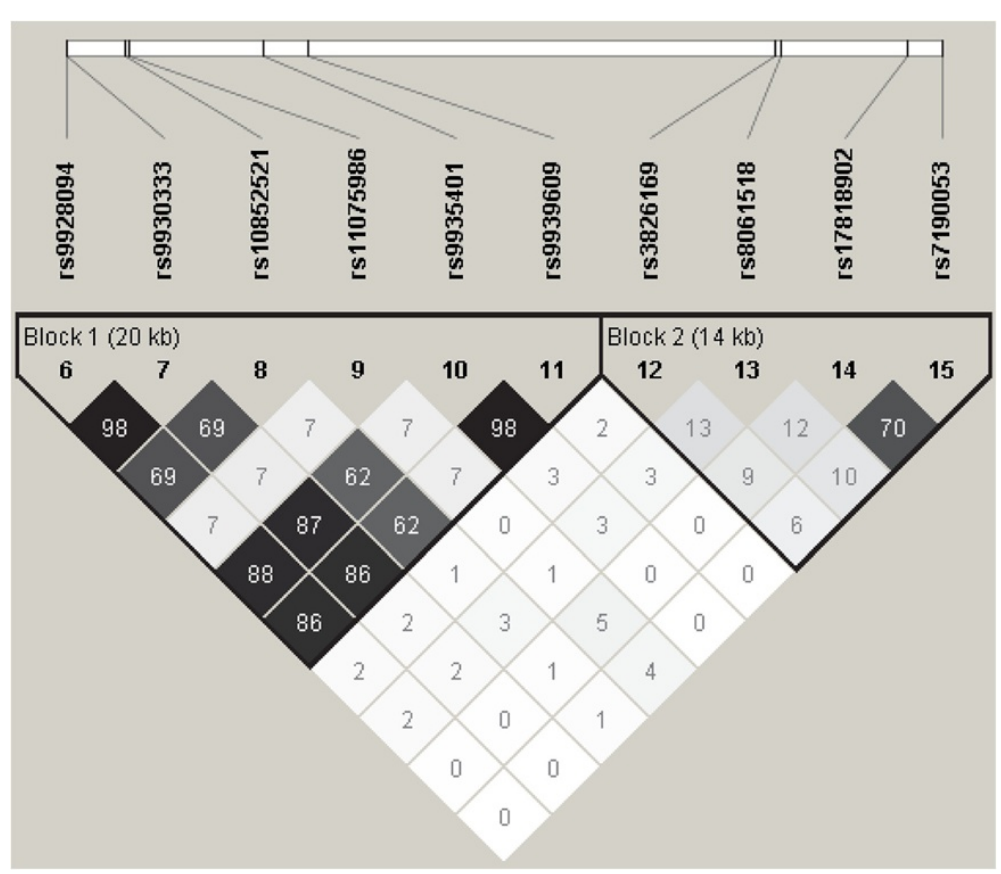

Figure 1 Linkage disequilibrium structure in terms of $r^{2}$ of 10 SNPs genotyped, representing two well-defined haplo-blocks.

Zimmerman et al. [33] included other biomarkers, such as IL-6, IL-1 $\beta$, IL-10, IL-18 or TNF $\alpha$, and found no significant association in line with our findings. The fact that the majority of studies has failed to associate the FTO variants with obesity related trait, indicates that this gene is in a very close relationship with obesity and fat mass. The potential effect on the obesity-related traits seems indirect and dependent on BMI changes.

Table 5 Association analysis of obesity with FTO haplotypes combinations

\begin{tabular}{|c|c|c|c|c|c|}
\hline \multirow[t]{2}{*}{ Haplotypes } & \multicolumn{3}{|c|}{ Frequency } & \multirow[b]{2}{*}{ OR $(95 \% \mathrm{Cl})$} & \multirow[b]{2}{*}{$\mathbf{P}$} \\
\hline & All & Case & Control & & \\
\hline \multicolumn{6}{|l|}{ Block 1} \\
\hline ATTCGT & 0.437 & 0.401 & 0.481 & Reference & \\
\hline GGCCAA & 0.425 & 0.483 & 0.355 & $1.26(1.64-2.14)$ & $5.11 \times 10^{-5}$ \\
\hline ATCGGT & 0.080 & 0.073 & 0.087 & $0.99(0.63-1.57)$ & 0.413 \\
\hline GGCCGT & 0.025 & 0.019 & 0.031 & $0.65(0.29-1.45)$ & 0.133 \\
\hline \multicolumn{6}{|l|}{ Block 2} \\
\hline CATC & 0.223 & 0.237 & 0.207 & Reference & \\
\hline TGTC & 0.297 & 0.258 & 0.345 & $0.65(0.47-0.92)$ & 0.002 \\
\hline TATC & 0.212 & 0.225 & 0.196 & $1.02(0.71-1.45)$ & 0.231 \\
\hline TAGT & 0.179 & 0.193 & 0.163 & $1.02(0.70-1.51)$ & 0.217 \\
\hline TAGC & 0.053 & 0.063 & 0.041 & $1.29(0.70-2.38)$ & 0.143 \\
\hline
\end{tabular}

Block 1 involves SNPs: rs9928094, rs9930333, rs10852521, rs11075986, rs9935401, and rs9939609, Overall p value: $8.72 \times 10^{-4}$. Block 2 involves SNPs: rs3826169, rs8061518, rs17818902, and rs7190053. Overall p value: 0.026 . OR: odds ratio; $\mathrm{Cl}$ : confidence interval.
The association of the variant rs9939609 with MS has been shown in European [25] and mixed population [34]. Furthermore, rs1421085 which is highly correlated $\left(\mathrm{LD} \mathrm{r}^{2}=0.931\right)$ [3] with rs9939609, was associated with MS in an independent study [35]. On the contrary, rs9939609 was not associated with MS in Japanese [36] or Indian [37] population. As we discussed previously, some studies showed an association with cardiovascular markers, also related to MS, whereas others did not. The same is true for glucose metabolism and BP; some studies found a significant association with glucose and insulin [38] and others, in concordance with our work, did not [26-28]. In the case of BP, neither Freathy et al. [25] nor Kring et al. [26] observed an association; however, Pausova et al. [39] did otherwise with systolic BP in adolescents. Moreover, a recent genome-wide scan identified an association between rs9930333 (SNP in LD with rs9939609) and BP in adolescents [40]. In our study population, the percentage of MS in the obese group was $9.8 \%$, and we did not observe association of any SNPs with MS (data not shown). These results could be due to the small size of the study population as it has been reported that sample sizes of at least 12000 are required to observe association between FTO genotype and metabolic traits that are secondary to changes in BMI [25]. In addition, the lack of association found in our study might also be due to the age of our population (mean of 9.6 years), because it has been demonstrated that rs9939609 showed changes in its association with BMI as 
children grow up and enter into youth. One study showed no association at age of 4 years, but this association became increasingly stronger at ages 7-11 years [41]; other study, observed a biphasic change in the associations of this variant with BMI, strengthening with age up to a peak at age 20 years, and then weakening with increasing adult age [42].

Beyond the replication of association found in SNPs within the first intron, we interestingly identified a novel SNP (rs8061518), localized in the third intron of the gene about 40-60 kb away from the originally reported SNPs in the first intron, which was associated with reduced risk of obesity. This SNP was also negatively associated with some MS traits, leptin and aPAI-1. Although our results should be validated in larger independent studies, we used the publicly available GIANT consortium published data [43], and we found that the rs8061518 was associated with BMI with a $\mathrm{p}=0.0149$ in a population of 123844 European descent adults, and the association effect was in the same direction that we observed in our population. Only one previous study in Sorb population [44] has demonstrated that other SNPs in the same region of the gene were associated with reduced risk of obesity, among them the strongest effect on BMI values was associated with rs17818902. However, in the present study we did not confirm this association, in concordance with Dlouha et al. who genotyped this SNP in a group of 2559 unrelated European descent population [45]. In addition, no association of this variant with BMI was found in the GIANT consortium ( $\mathrm{p}=$ 0.206). One possible explanation of this is that the rs17818902 represents a second mutational event segregating in the Sorbian population.

The haplotype analysis shows that a unique haplotype TGTC (haplo-block 2) with the minor allele of the rs8061518 exhibited a decreased risk for obesity. These results indicate that there may be other regions beyond the intron 1 in the FTO gene associated with being lean or obese that needs to be studied.

The association of the FTO gene with leptin has also been studied. Labayen et al. [9], Rutters et al. [14], and Zimmerman et al. [33] found a positive association of the rs9939609 with leptin, whereas Do et al. [23] did it with the rs17817449; however, this association was abolished after adjusting for BMI. In our population, we did not observe an association in the case of the rs9939609, but interestingly we found a negative association with rs8061518 that remains significant after adjustment by sex, age and BMI. Leptin, a hormone produced mainly by the white adipose tissue, is clearly associated with the total amount of body fat and secondly with BMI. It participates in the regulation of a range of biological functions and process including energy intake and expenditure, neuroendocrine system, autonomic function, reproduction, and glucose homeostasis $[46,47]$. As this hormone is involved in three different pathways [47], its mechanisms of action act through different signalling pathways. Studies in animals [48] and humans [49] have demonstrated that it can exert its action independently of obesity. Then, it might be the case in the association of this adipokine with the rs8061518; however, further studies are needed to clarify this aspect.

Our study has several strengths and limitations to highlight. The strengths are the high quantity of biomarkers measured in the children, the genotyping of several SNPs along the FTO gene and the way of SNPs election. We selected part of the SNPs from a group with a MAF higher than 0.05 in the European descent population from the NCBI database to make an association case-control study as complementary strategy to GWAS in the identification of gene variants associated with obesity and its comorbidities. The limitations are first, the sample size of the current investigation is relatively small for a genetic association with metabolic traits. Second, there are a number of environmental factors known to influence body weight in children, which were not taken into account in this study. Furthermore, information on body composition, nutrition and physical activity, which are strongly associated factors with body-weight regulation, was not available. Fat composition data could be valuable for the adjustment of the association of rs8061518 with leptin in order to clarify the role of the genetic variant on obesity.

\section{Conclusion}

We confirmed the previously reported association of genetic variability in intron 1 of the FTO gene with the risk of obesity and without association with other related traits of inflammation and CVD risk biomarkers. We also have observed strong statistical evidence for an association of rs8061518 in intron 3 of the gene with decreased risk of obesity and low concentration of leptin. Additionally, both studied haplo-blocks, that included the significant associated SNPs, were also associated with the risk of obesity. Our findings provide qualified support for the notion that a detailed examination of the third intron region of the FTO gene in larger independent studies should provide valuable clues to the molecular mechanisms by which sequence variation in this region affects clinical phenotypes.

\section{Additional file}

\footnotetext{
Additional file 1: Table S1. Description of the genotyped polymorphisms. Table S2. Association of rs9928094 with biomarkers of inflammation and cardiovascular disease risk in obese children. Table S3. Association of rs9930333 with biomarkers of inflammation and cardiovascular disease risk in obese children. Table S4. Association of rs9935401 with biomarkers of inflammation and cardiovascular disease risk in obese children.
} 


\section{Abbreviations}

ApoA1: Apolipoprotein A1; ApoB: Apolipoprotein B; BMl: Body mass index $\mathrm{BP}$ : Blood pressure; Cl: Confidence interval; $\mathrm{CV}$ : Coefficient of variation; CVD: Cardiovascular diseases; FTO: Fat mass and obesity associated; HDL-c: High-density lipoprotein-cholesterol; HOMA-IR: Homeostatic model assessment of insulin resistance; IL: Interleukin; LD: Linkage disequilibrium; LDL-c: Density lipoprotein-cholesterol; MAF: Minor allele frequency; MMP-9: Matrix metalloproteinase-9; MPO: Myeloperoxidase; MS: Metabolic syndrome; OR: Odds ratio; PAI-1: Plasminogen activator inhibitor-1; SE-selectin: Soluble endothelial selectin; sICAM-1: Soluble intercellular adhesion molecule 1; SNP: Single nucleotide polymorphism; TNF-a: Tumour necrosis factor alpha; WC: Waist circumference.

\section{Competing interests}

The authors declare that they have no competing interests.

\section{Authors' contributions}

Conception and design of the study: AG, CMA. Collection of the children's and acquisition of the data: MG-C, RL, RT, RC. Biomarkers analysis: JO. Genotyping analysis: DF-O. Statistical analysis: JO, AIR, DF-O. Interpretation of the data and drafting the manuscript: JO, CMA. Critical revision of the manuscript: JO, AG, CMA. All authors have read and approved the final version of the manuscript.

\section{Acknowledgements}

This work was supported by Plan Nacional de Investigación Científica, Desarrollo e Innovación Tecnológica (I + D + I), Instituto de Salud Carlos III-Fondo de Investigación Sanitaria (PI020826, P1051968), Consejería de Innovación y Ciencia, Junta de Andalucía (P06-CTS 2203) and Ministerio de Universidades y Tecnología, Instituto de Salud Carlos III, Fondo de Investigaciones Sanitarias, RETIC (Red SAMID RD08/0072/0028).

\section{Author details}

'Department of Biochemistry and Molecular Biology II, Faculty of Pharmacy Institute of Nutrition and Food Technology, University of Granada, Granada, Spain. ${ }^{2}$ Paediatric Research and Metabolism Unit, Reina Sofía University Hospital, Maimonides Institute for Biomedical Research (IMIBIC), Córdoba, Spain. ${ }^{3}$ Unit of Investigation in Nutrition, Growth and Human Development of Galicia, Paediatric Department, Clinic University Hospital of Santiago, University of Santiago de Compostela, Galicia, Spain. ${ }^{4}$ Progenika Biopharma S.A, Building 504, Zamudio Technologic Park. Derio, Bizkaia, Spain.

Received: 28 March 2013 Accepted: 25 November 2013

Published: 1 December 2013

\section{References}

1. Pietrobelli A, Espinoza MC, De Cristofaro P: Childhood obesity: looking into the future. Angiology 2008, 59:30S-33S

2. Abrams $P$, Levitt Katz LE: Metabolic effects of obesity causing disease in childhood. Curr Opin Endocrinol Diabetes Obes 2011, 18:23-27.

3. Frayling TM, Timpson NJ, Weedon MN, Zeggini E, Freathy RM, Lindgren CM, Perry JR, Elliott KS, Lango H, Rayner NW, Shields B, Harries LW, Barrett JC, Ellard S, Groves CJ, Knight B, Patch AM, Ness AR, Ebrahim S, Lawlor DA, Ring SM, Ben-Shlomo Y, Jarvelin MR, Sovio U, Bennett AJ, Melzer D, Ferrucci L, Loos RJ, Barroso I, Wareham NJ, et al: A common variant in the FTO gene is associated with body mass index and predisposes to childhood and adult obesity. Science 2007, 316:889-894

4. Dina C, Meyre D, Gallina S, Durand E, Körner A, Jacobson P, Carlsson LM, Kiess W, Vatin V, Lecoeur C, Delplanque J, Vaillant E, Pattou F, Ruiz J, Weill J, Levy-Marchal C, Horber F, Potoczna N, Hercberg $S$, Le Stunff C, Bougnères $P$, Kovacs $P$, Marre M, Balkau B, Cauchi S, Chèvre JC, Froguel P: Variation in FTO contributes to childhood obesity and severe adult obesity. Nat Genet 2007, 39:724-726.

5. Scuteri A, Sanna S, Chen WM, Uda M, Albai G, Strait J, Najiar S, Nagaraja R, Orrú M, Usala G, Dei M, Lai S, Maschio A, Busonero F, Mulas A, Ehret GB, Fink AA, Weder AB, Cooper RS, Galan P, Chakravarti A, Schlessinger D, Cao A, Lakatta E, Abecasis GR: Genome-wide association scan shows genetic variants in the FTO gene are associated with obesity-related traits. PLoS Genet 2007, 3:1200-1210.

6. Peng S, Zhu Y, XU F, Ren X, Li X, Lai M: FTO gene polymorphisms and obesity risk: a meta-analysis. BMC Med 2011, 9:71.
7. Hinney A, Nguyen $\Pi$ T, Scherag A, Friedel S, Brönner G, Müller TD, Grallert H, Illig T, Wichmann HE, Rief W, Schäfer H, Hebebrand J: Genome wide association (GWA) study for early onset extreme obesity supports the role of fat mass and obesity associated gene (FTO) variants. PLoS One 2007, 2:e1361

8. Hunt SC, Stone S, Xin Y, Scherer CA, Magness CL, ladonato SP, Hopkins PN Adams TD: Association of the FTO gene with BMI. Obesity 2008, 16:902-904.

9. Labayen I, Ruiz JR, Ortega FB, Gottrand F, Huybrechts I, Dallongeville J, Widhalm K, Ferrari M, Buyken A, Kersting M, Moschonis G, Turck D, Gómez S, Sjostrom M, Meirhaeghe A, Moreno LA: Body size at birth modifies the effect of fat mass and obesity associated (FTO) rs9939609 polymorphism on adiposity in adolescents: the healthy lifestyle in Europe by nutrition in adolescence (HELENA) study. Br J Nutr 2008, 107:1498-1504.

10. Xi B, Shen $Y$, Zhang $M$, Liu $X$, Zhao $X$, Wu L, Cheng H, Hou D, Lindpaintner K, Liu L, Mi J, Wang X: The common rs9939609 variant of the fat mass and obesity-associated gene is associated with obesity risk in children and adolescents of Beijing. China. BMC Med Genet 2010, 11:107.

11. Fang H, Li Y, Du S, Hu X, Zhang Q, Liu A, Ma G: Variant rs9939609 in the FTO gene is associated with body mass index among Chinese children. BMC Med Genet 2010, 11:136.

12. Hassanein MT, Lyon HN, Nguyen TT, Akylbekova EL, Waters K, Lettre G, Tayo B, Forrester T, Sarpong DF, Stram DO, Butler JL, Wilks R, Liu J, Le Marchand L, Kolonel LN, Zhu X, Henderson B, Cooper R, McKenzie C, Taylor HA Jr, Haiman CA, Hirschhorn JN: Fine mapping of the association with obesity at the FTO locus in African-derived populations. Hum Mol Genet 2010, 19:2907-2916.

13. Wing MR, Ziegler J, Langefeld CD, Ng MC, Haffner SM, Norris JM, Goodarzi MO, Bowden DW: Analysis of FTO gene variants with measures of obesity and glucose homeostasis in the IRAS Family Study. Hum Genet 2009, 125:615-626.

14. Rutters F, Nieuwenhuizen AG, Bouwman F, Mariman E, Westerterp-Plantenga MS: Associations between a single nucleotide polymorphism of the FTO Gene (rs9939609) and obesity-related characteristics over time during puberty in a Dutch children cohort. J Clin Endocrinol Metab 2009, 96:E939-E942.

15. Liu G, Zhu H, Lagou V, Gutin B, Stallmann-Jorgensen IS, Treiber FA, Dong Y, Snieder H: FTO variant rs9939609 is associated with body mass index and waist circumference, but not with energy intake or physical activity in European- and African-American youth. BMC Med Genet 2010, 11:57.

16. Grant SF, Li M, Bradfield JP, Kim CE, Annaiah K, Santa E, Glessner JT, Casalunovo T, Frackelton EC, Otieno FG, Shaner JL, Smith RM, Imielinski M, Eckert AW, Chiavacci RM, Berkowitz RI, Hakonarson H: Association analysis of the FTO gene with obesity in children of Caucasian and African ancestry reveals a common tagging SNP. PLOS One 2008, 3:e1746.

17. Cole TJ, Bellizzi MC, Flegal KM, Dietz WH: Establishing a standard definition for child overweight and obesity worldwide: international survey. BMJ 2000, 320:1240-1243.

18. González JR, Armengol L, Solé X, Guinó E, Mercader JM, Estivill X, Moreno V: SNPassoc: an $\mathrm{R}$ package to perform whole genome association studies. Bioinformatics 2007, 23:644-645.

19. Cecil JE, Tavendale R, Watt $P$, Hetherington MM, Palmer CAN: An obesity-associated FTO gene variant and increased energy intake in children. New Eng J Med 2008, 359:2558-2566.

20. Heard-Costa NL, Zillikens MC, Monda KL, Johansson A, Harris TB, Fu M, Haritunians T, Feitosa MF, Aspelund T, Eiriksdottir G, Garcia M, Launer L, Smith AV, Mitchell BD, McArdle PF, Shuldiner AR, Bielinski SJ, Boerwinkle E, Brancati F, Demerath EW, Pankow JS, Arnold AM, Chen YD, Glazer NL, McKnight B, Psaty BM, Rotter Jl, Amin N, Campbell H, Gyllensten U, et al: NRXN3 is a novel locus for waist circumference: a genome-wide association study from the CHARGE Consortium. PLoS Genet 2009, 5:e1000539.

21. Wang K, Li WD, Zhang CK, Wang Z, Glessner JT, Grant SF, Zhao H, Hakonarson $\mathrm{H}$, Price RA: A genome-wide association study on obesity and obesity-related traits. PLoS One 2011, 6:e18939.

22. Ruiz JR, Labayen I, Ortega FB, Legry V, Moreno LA, Dallongeville J, Martínez-Gómez D, Bokor S, Manios Y, Ciarapica D, Gottrand F, De Henauw S, Molnár D, Sjöström M, Meirhaeghe A, HELENA Study Group: Attenuation of the effect of the FTO rs9939609 polymorphism on total and central body fat by physical activity in adolescents: the HELENA study. Arch Pediatr Adolesc Med 2010, 164:328-333.

23. Do R, Bailey SD, Desbiens K, Belisle A, Montpetit A, Bouchard C, Pérusse L, Vohl MC, Engert JC: Genetic variants of FTO influence adiposity, insulin sensitivity, leptin levels, and resting metabolic rate in the Quebec family study. Diabetes 2008, 57:1147-1150. 
24. Belo VA, Souza-Costa DC, Luizon MR, Lanna CM, Carneiro PC, Izidoro-Toledo TC, Ferraz KC, Tanus-Santos JE: Matrix metalloproteinase-9 genetic variations affect MMP-9 levels in obese children. Int J Obes 2011, 36:69-75.

25. Freathy RM, Timpson NJ, Lawlor DA, Pouta A, Ben-Shlomo Y, Ruokonen A, Ebrahim S, Shields B, Zeggini E, Weedon MN, Lindgren CM, Lango H, Melzer D, Ferrucci L, Paolisso G, Neville MJ, Karpe F, Palmer CN, Morris AD, Elliott P, Jarvelin MR, Smith GD, McCarthy MI, Hattersley AT, Frayling TM: Common variation in the FTO gene alters diabetes-related metabolic traits to the extent expected given its effect on BMI. Diabetes 2008, 57:1419-1426.

26. Kring Sl, Holst C, Zimmermann E, Jess T, Berentzen T, Toubro S, Hansen T, Astrup A, Pedersen O, Sørensen TI: FTO gene associated fatness in relation to body fat distribution and metabolic traits throughout a broad range of fatness. PloS One 2008, 3:e2958.

27. Müller TD, Hinney A, Scherag A, Nguyen TT, Schreiner F, Schäfer $H$ Hebebrand J, Roth CL, Reinehr T: Fat mass and obesity associated' gene (FTO): no significant association of variant rs9939609 with weight loss in a lifestyle intervention and lipid metabolism markers in German obese children and adolescents. BMC Med Genet 2008, 9:85.

28. Zavattari P, Loche A, Pilia S, Ibba A, Moi L, Guzzetti C, Casini MR: Loche S: rs9939609 in the FTO gene is associated with obesity but not with several biochemical parameters in Sardinian obese children. Ann Hum Genet 2011, 75:648-654.

29. Ahmad T, Chasman DI, Mora S, Paré G, Cook NR, Buring JE, Ridker PM, Lee IM: The fat-mass and obesity-associated (FTO) gene, physical activity, and risk of incident cardiovascular events in white women. Am Heart J 2010, 160:1163-1169.

30. Lappalainen T, Kolehmainen M, Schwab US, Tolppanen AM, Stančáková A Lindström J, Eriksson JG, Keinänen-Kiukaanniemi S, Aunola S, Ilanne-Parikka P, Herder C, Koenig W, Gylling H, Kolb H, Tuomilehto J, Kuusisto J, Uusitupa M: Finnish diabetes prevention study group: association of the FTO gene variant (rs9939609) with cardiovascular disease in men with abnormal glucose metabolism-the Finnish diabetes prevention study. Nutr Metab Cardiovasc Dis 2011, 21:691-698.

31. Timpson NJ, Nordestgaard BG, Harbord RM, Zacho J, Frayling TM, Tybjærg-Hansen A, Smith GD: C-reactive protein levels and body mass index: elucidating direction of causation through reciprocal Mendelian randomization. Int J Obes 2011, 35:300-308.

32. Fisher $\mathrm{E}$, Schulze MB, Stefan $\mathrm{N}$, Häring HU, Döring F, Joost HG, Al-Hasani $\mathrm{H}$, Boeing $\mathrm{H}$, Pischon T: Association of the FTO rs9939609 single nucleotide polymorphism with C-reactive protein levels. Obesity 2009, 17:330-334

33. Zimmermann E, Skogstrand K, Hougaard DM, Astrup A, Hansen T, Pedersen O, Sørensen TI, Jess T: Influences of the common FTO rs9939609 variant on inflammatory markers throughout a broad range of body mass index. PLoS One 2011, 6:e15958.

34. Al-Attar SA, Pollex RL, Ban MR, Young TK, Bjerregaard P, Anand SS, Yusuf S, Zinman B, Harris SB, Hanley AJ, Connelly PW, Huff MW, Hegele RA: Association between the FTO rs9939609 polymorphism and the metabolic syndrome in a non-Caucasian multiethnic sample. Cardiovasc Diabetol 2008, 7:5.

35. Attaoua R, Ait El Mkadem S, Lautier C, Kaouache S, Renard E, Brun JF, Fedou C, Gris JC, Bringer J, Grigorescu F: Association of the FTO gene with obesity and the metabolic syndrome is independent of the IRS-2 gene in the female population of Southern France. Diabetes Metab 2009, 35:476-483.

36. Shimaoka I, Kamide K, Ohishi M, Katsuya T, Akasaka H, Saitoh S, Sugimoto K, Oguro R, Congrains A, Fujisawa T, Shimamoto K, Ogihara T, Rakugi H: Association of gene polymorphism of the fat-mass and obesity-associated gene with insulin resistance in Japanese. Hypertens Res 2010, 33:214-218.

37. Ranjith N, Pegoraro RJ, Shanmugam R: Obesity-associated genetic variants in young Asian Indians with the metabolic syndrome and myocardial infarction. Cardiovasc J Afr 2011, 22:25-30.

38. Luczynski W, Zalewski G, Bossowski A: The association of the FTO rs9939609 polymorphism with obesity and metabolic risk factors for cardiovascular diseases in Polish children. J Physiol Pharmacol 2012, 63:241-248.

39. Pausova Z, Syme C, Abrahamowicz M, Xiao Y, Leonard GT, Perron M, Richer L, Veillette S, Smith GD, Seda O, Tremblay J, Hamet P, Gaudet D, Paus T: A common variant of the FTO gene is associated with not only increased adiposity but also elevated blood pressure in French Canadians. Circ Cardiovasc Genet 2009, 2:260-269.

40. Melka MG, Bernard M, Mahboubi A, Abrahamowicz M, Paterson AD, Syme C, Lourdusamy A, Schumann G, Leonard GT, Perron M, Richer L, Veillette S,
Gaudet D, Paus T, Pausova Z: Genome-wide scan for Loci of adolescent obesity and their relationship with blood pressure. J Clin Endocrinol Metab 2011, 97:E145-E150.

41. Haworth CM, Carnell S, Meaburn EL, Davis OS, Plomin R, Wardle J: Increasing heritability of BMI and stronger associations with the FTO gene over childhood. Obesity 2008, 16:2663-2668.

42. Hardy R, Wills AK, Wong A, Elks CE, Wareham NJ, Loos RJ, Kuh D, Ong KK: Life course variations in the associations between FTO and MC4R gene variants and body size. Hum Mol Genet 2010, 19:545-552.

43. Speliotes EK, Willer CJ, Berndt SI, Monda KL, Thorleifsson G, Jackson AU, Lango Allen H, Lindgren CM, Luan J, Mägi R, Randall JC, Vedantam S, Winkler TW, Qi L, Workalemahu T, Heid IM, Steinthorsdottir V, Stringham HM, Weedon MN, Wheeler E, Wood AR, Ferreira T, Weyant RJ, Segrè AV, Estrada K, Liang L, Nemesh J, Park JH, Gustafsson S, et al: Association analyses of 249,796 individuals reveal 18 new loci associated with body mass index. Nat Genet 2010, 42:937-948.

44. Tönjes A, Zeggini E, Kovacs P, Böttcher $Y$, Schleinitz D, Dietrich K, Morris AP, Enigk B, Rayner NW, Koriath M, Eszlinger M, Kemppinen A, Prokopenko I, Hoffmann K, Teupser D, Thiery J, Krohn K, McCarthy MI, Stumvoll M: Association of FTO variants with BMI and fat mass in the self-contained population of Sorbs in Germany. Eur J Hum Genet 2010, 18:104-110.

45. Dlouha D, Adamkova $V$, Lanska $V$, Hubacek JA: Lack of association between a new tag SNP in the FTO gene and BMI in Czech-Slavonic population. Eur J Hum Genet 2010, 18:1274.

46. Varela L, Horvath TL: Leptin and insulin pathways in POMC and AgRP neurons that modulate energy balance and glucose homeostasis. EMBO Rep 2012, 13:1079-1086.

47. Paz-Filho G, Mastronardi C, Franco CB, Wang KB, Wong ML, Licinio J: Leptin: molecular mechanisms, systemic pro-inflammatory effects, and clinical implications. Arq Bras Endocrinol Metabol 2012, 56:597-607.

48. Berglund ED, Vianna CR, Donato J Jr, Kim MH, Chuang JC, Lee CE, Lauzon DA, Lin P, Brule $\sqcup$, Scott MM, Coppari R, Elmquist JK: Direct leptin action on POMC neurons regulates glucose homeostasis and hepatic insulin sensitivity in mice. J Clin Invest 2012, 122:1000-1009.

49. Esteghamati A, Khalilzadeh O, Anvari M, Rashidi A, Mokhtari M, Nakhjavani M: Association of serum leptin levels with homeostasis model assessment-estimated insulin resistance and metabol-ic syndrome: the key role of central obesity. Metab Syndr Relat Disord 2009, 7:447-452.

doi:10.1186/1471-2350-14-123

Cite this article as: Olza et al:: Influence of FTO variants on obesity, inflammation and cardiovascular disease risk biomarkers in Spanish children: a case-control multicentre study. BMC Medical Genetics 2013 14:123.

\section{Submit your next manuscript to BioMed Central and take full advantage of:}

- Convenient online submission

- Thorough peer review

- No space constraints or color figure charges

- Immediate publication on acceptance

- Inclusion in PubMed, CAS, Scopus and Google Scholar

- Research which is freely available for redistribution 